\title{
Desarrollo de cohortes y parámetros poblacionales de la araña del rincón Loxosceles laeta
}

\author{
Mauricio Canals y Rigoberto Solís
}

\section{Development and population parameters of cohorts of the Chilean recluse spider Loxosceles laeta}

Background: Despite the abundant eco-epidemiological knowledge of the Chilean reclusive spider, Loxosceles laeta, which causes all forms of loxoscelism in Chile, the main characteristics of this species its stages of development remains poorly known especially in the medical area. Objective: In this study we address these issues with the goal of providing clear images of the development of this species and for the first time on population projections as well as the relationship between mature and immature instars, useful data for the control and prevention of accidental bites. Results: We found that L. laeta is an r-selected species, with $R_{0}=2.1$, a generation time of $G=2.1$ years, with a concentration of the reproductive value of females between the first and second year of life. We determined the average sizes and development times of all instars. The first vary between $2.3 \mathrm{~mm}$ at birth and about $13 \mathrm{~mm}$ at adulthood. The total development time was about 1 year. Discussion: The population projection by Leslie matrix suggested great capacity for growth and dispersal with clear seasonal population fluctuations associated with reproduction. It also showed that the proportion of immature varied seasonally between 80 and $90 \%$, which means that a house with three or four visible adult spiders actually has a population between 20 and 40 spiders in total.

Key words: Loxoscelism, Loxosceles laeta, development, population.

Palabras clave: Loxoscelismo, Loxosceles laeta, desarrollo, poblaciones.

\section{Introducción}

$\mathrm{E}$ 1 loxoscelismo es el cuadro clínico generado por arañas del género Loxosceles, y se ha registrado en todos los continentes. En América se han registrado casos de loxoscelismo atribuidos a Loxosceles laeta en el cono Sur, L. reclusa en Estados Unidos de América (E.U.A.) y México, y L. arizonica en E.U.A.; $L$. gaucho en Argentina, $L$. intermedia en Brasil y $L$. rufescens, $L$. spadicea en zonas mediterráneas ${ }^{1}$. En Perú el loxoscelismo se atribuye a $L$. laeta y $L$. rufipes ${ }^{2}$. Aunque en Chile desde 1878 se reporta la "mancha necrótica" por picadura de araña, fue Macchiavelo (1937) quien informó y demostró que numerosos casos de accidentes cutáneonecróticos asociados a trastornos viscerales y hemolíticos eran consecuencia de la mordedura de L. laeta ${ }^{4}$. Veinte años después Atkins demostró la patogenicidad de $L$. reclusa en E.U.A. ${ }^{5}$.

En Sudamérica se han reportado cuadros necróticos producidos por las arañas lobo (Lycosidae) causados por Lycosa raptoria, L. erytrognatha, L. pampeana y $L$. thorelli. En Chile existen numerosas especies de la familia Lycosidae y del género Lycosa, revisados por Casanueva $(1980)^{6}$, pero no existen reportes de daño por esta araña, salvo una cita aislada de la posible responsabilidad de $L$. murina en lesiones necróticas cutáneas ${ }^{7}$. Así, en Chile sólo se ha responsabilizado a $L$. laeta de todos los casos de aracnoidismo necrótico (loxoscelismo) ${ }^{8-13}$. Este ocurre fundamentalmente por la presencia de la enzima esfingomielinasa $\mathrm{D}^{14}$ que también está presente en otras arañas del género Sicarius, que pertenecen a la misma familia (Sicariidae). Aunque en Chile existen ocho especies de este género descritas ${ }^{15}$, como las comunes Sicarius crustosus y $S$. terrosus, no se han reportado accidentes con estas arañas ${ }^{12}$.

El loxoscelismo es un importante problema de salud pública en América, especialmente en el cono Sur y en Chile ${ }^{14}$. Así, por ejemplo en 2005, el centro de atención toxicológica de la Pontificia Universidad Católica de Chile atendió 2.831 llamados telefónicos sobre pacientes con sospecha de loxoscelismo, confirmándose 287 de éstos ${ }^{16}$. Sólo en el Hospital Clínico de la Universidad de Chile se siguieron y confirmaron 250 casos hospitalizados entre 1955 y 2000, con 56 casos pediátricos ${ }^{9,10,17}$.

Existen dos cuadros clínicos principales asociados a loxoscelismo: loxoscelismo cutáneo con una frecuencia de $83 \%$ y que se caracteriza por daño local, dolor, edema, eritema e isquemia durante las primeras ocho horas, que progresa a la vasoconstricción e isquemia. Además se puede generar una mancha, con o sin ampollas, que poseen una coloración azul-violeta (mancha livedoide) de consistencia dura y centro deprimido. A partir de esta lesión se puede generar una ulceración y necrosis del área total. Aunque la necrosis es profunda, el cuadro es
Universidad de Chile, Santiago, Chile.

Facultad de Medicina, Departamento de Medicina (Oriente) (MC).

Facultad de Ciencias Veterinarias y Silvoagropecuarias, Departamento de Ciencias Biológicas Animales (RS).

Recibido: 5 de marzo de 2014 Aceptado: 17 de julio de 2014

Correspondencia a: Mauricio Canals Lambarri mcanals@uchile.cl 
local. En ocasiones puede existir una variante edematosa del cuadro ${ }^{8,14}$. Otro de los cuadros es el loxoscelismo cutáneo-visceral y es más grave que el anterior siendo letal en $22,2 \%$ de los niños y $19,1 \%$ de los adultos. Se caracteriza por tener un componente sistémico, en cual se caracteriza por fiebre, hemolisis, hematuria, hemoglobinuria e insuficiencia renal que se instala rápidamente, e ictericia y daño hepático dentro de las primeras $24 \mathrm{~h}^{8,9}$.

A pesar de que en Chile todo el aracnoidismo necrótico es atribuido a $L$. laeta, su biología sigue siendo poco conocida en el ambiente médico ${ }^{11-13,18}$. Loxosceles laeta es un araña errante solitaria crepuscular y nocturna asociada al hábitat doméstico, común en habitaciones humanas y en jardines de casas de la Zona Central de Chile ${ }^{19,20}$ encontrándose en el interior de los hogares habitualmente en esquinas oscuras, ropa de baño, grietas, pero también en ocasiones se pueden encontrar al aire libre. En estos ambientes halla su temperatura preferida entre 16 y $23^{\circ} \mathrm{C}$, mayor en las tardes, pudiendo tolerar temperaturas muy extremas entre -5 y $48{ }^{\circ} \mathrm{C}^{21}$. Su tolerancia a la desecación es la propia de una especie adaptada a ambientes xéricos ${ }^{22}$. $\mathrm{Su}$ color es pardo claro u oscuro, dependiendo del estado de desarrollo y del sexo. Los estados inmaduros y los machos son más claros. Tiene tres pares de ojos simples, dos laterales y uno anterior. Los machos presentan los pedipalpos más largos. La hembra adulta mide alrededor de 1 a $1,5 \mathrm{~cm}$ en su cuerpo, pudiendo llegar a una envergadura de $4,5 \mathrm{~cm}$ con las patas extendidas ${ }^{9}$. Construye pequeñas telas irregulares, donde habitualmente deja su ooteca. Teje una ooteca o capullo de aproximadamente 1 $\mathrm{cm}$, en la que se pueden encontrar entre 80 y 200 huevos, con un promedio de $90^{1}$. Su desarrollo una vez fuera de la ooteca, dura aproximadamente un año para llegar a adulto, pasando por tres estadios de desarrollo que se completan entre 9 a 12 mudas $^{23}$. Su longevidad es, en promedio, 696 días para machos que se han apareado, 1.155 días para machos vírgenes, 1.536 días para hembras fecundadas y 1.894 días para hembras vírgenes, aunque se reportan máximos de 4,5 a 4,8 años ${ }^{24-26}$.

$\mathrm{Su}$ veneno es de naturaleza proteica, compuesta por polipéptidos. Contiene hialuronidasa, levartenerol, y esfingomielinasa, las que tienen acción citotóxica, coagulante, proteolítica y hemolítica ${ }^{27}$.

La epidemiologia de los accidentes de loxoscelimo coincide con los hábitos nocturnos, ya que éstos son más frecuentes en horas del crepúsculo y la noche. Además los datos epidemiológicos sugieren mayores poblaciones y actividad en el verano ${ }^{8-10,17}$. Los accidentes ocurren predominantemente en primavera y verano (75\%), y preferentemente entre las 22 y las $7 \mathrm{~h}(75 \%)$ y en general dentro de los dormitorios $(69,6 \%)^{17}$. Las circunstancias más habituales en que ocurre la mordedura son mientras se duerme, al vestirse, al secarse con toallas y durante el juego en niños ${ }^{17}$. Sólo en alrededor de un $10 \%$ de los casos la araña es capturada y llevada para identificación ${ }^{8-10,17}$.

Con referencia a la dieta, en Chile se ha reportado que se alimenta de moscas, polillas y otros pequeños artrópo$\operatorname{dos}^{8-10,17,19,23,28}$. El único estudio sistemático conducido en doce telas reportó las siguientes presas: moscas 30,6\%, escarabajos $20,0 \%$, hormigas $13,3 \%$, isópodos $12,0 \%$, otras arañas $8,0 \%$, polillas $5,3 \%$, milipedos $4 \%$, tijeretas $2,7 \%$, mosquitos $2,7 \%$, grillos $1,3 \%$ y una cantidad no cuantificada de ácaros ${ }^{29}$. Su potencial depredador, Scytodes globula, es una araña solitaria que pertenece a la familia Scytodidae, que incluye arañas conocidas como arañas escupidoras, de reconocidos hábitos aracnofágicos ${ }^{21,22,30-33}$.

A pesar de todo este conocimiento eco-epidemiológico, L. laeta sigue siendo una especie poco conocida en el ambiente médico, especialmente las características y tiempos de sus estadios de desarrollo, que aunque descritos en forma taxonómica ${ }^{24,34-36}$ contienen pocos aspectos útiles para la práctica médica habitual. En este estudio abordamos estos aspectos con el objetivo de aportar imágenes claras del desarrollo de esta especie y por primera vez de sus características y proyecciones poblacionales y de la relación entre estados maduros e inmaduros, datos útiles para el control y prevención de los accidentes por mordedura.

\section{Material y Métodos}

Se capturaron y mantuvieron tres hembras adultas de la especie $L$. laeta con sus respectivos capullos. Cada una de estas hembras y sus capullos se introdujo en una caja de plástico hermética de $35 \times 25 \times 20 \mathrm{~cm}$ a temperatura ambiente de laboratorio, similar a cualquier casa de Santiago. Se mantuvieron estas arañas hasta el nacimiento de las arañuelas. A partir del nacimiento de las arañas (día 0) se contaron las arañas cada dos semanas, registrando las vivas, muertas y las mudas, retirando estas últimas. El seguimiento total fue de 450 días. Se determinó el estado de las sobrevivientes observando y contando las mudas y también por las características morfológicas de las arañuelas (siguiendo a Galiano 1967) ${ }^{34}$. Se fotografiaron a $50 \mathrm{~cm}$ de distancia instalando como referencia una marca de dimensiones conocidas. Se midió la temperatura y humedad relativa dentro de la caja con un Psicrómetro digital AZ8723 con sensor remoto. A cada cohorte se le administró con igual frecuencia un aporte constante de 10 larvas de Tenebrio molitor (escarabajo de la harina). Estas larvas varían de tamaño a medida que crecen por lo que se cuidó de administrar larvas de un tamaño manejable para las arañuelas (que no superara más de tres veces el tamaño de las arañuelas). Junto con el aporte de las larvas en cada ocasión se administraron 10 gotas de agua en una esquina para aportar humedad. Las arañuelas fueron mantenidas inicialmente con su madre, siendo ésta retirada al día 90. Durante cada inspección las arañas eran observadas 
durante $20 \mathrm{~min}$, registrando y fotografiando actividades tales como depredación y canibalismo. Además se mantuvieron arañas adultas de ambos sexos, alimentadas con larvas de Tenebrio molitor.

Se contaron las arañas en cada estado de desarrollo y se determinó la proporción de individuos en cada estado $(P)$ en función del tiempo, determinando el tiempo al cual $50 \%$ de los individuos cambiaba de estado $\left(T_{50}\right)$ mediante el modelo de probitos: probito $(P)=b_{0}+b_{l} t$, donde probito $(P)=z_{P}+5$ y $z_{P}$ es el valor de $z$ en una distribución normal $(0,1)$ para un valor particular $\mathrm{P}^{37}$.

En cada cohorte se registró el número de arañas $(N)$ y en las fotografías se midió el largo corporal (cefalotórax + abdomen) en el eje longitudinal de todas las arañas cuya posición lo permitía, sin incluir aquellas que estaban en posición oblicua o en posiciones que impidieran su medición, con un máximo de 20. La medida se realizó con el software Image y fue comparada con la marca de referencia, la que era re-medida en cada ocasión.

Se caracterizó el tamaño de cada estado de desarrollo de cada araña (promedio \pm desviación estándar). Con la variación en el número de arañuelas durante el desarrollo de las tres cohortes en conjunto se construyó la tabla de vida, considerando que las hembras de esta especie tienen una longevidad máxima de 4,5 años, y que una vez alcanzada la adultez (al año) la sobrevivencia $(p x)$ es prácticamente completa $(\approx 1)$ durante dos años, para descender sólo en el último período (un año y medio). Se consideraron 18 intervalos etarios con una duración $\mathrm{t}=$ 0,25 años. Las fecundidades $(m x)$ fueron consideradas como el valor promedio de arañuelas por saco, considerando sólo la fracción hembra de la población y considerando reproducción una vez al año, concentrada sólo en la etapa adulta. En la tabla de vida se siguió una metodología previamente establecida ${ }^{37-41}$ considerando: la proporción de sobrevivientes a la edad $\mathrm{x}(l x)$, la proporción de muertos $(d x)$, la probabilidad de muerte $(q x)$, la sobrevivencia $(p x=1-q x)$ y a partir de estos valores se calculó la tasa reproductiva neta $\left(R_{0}=\sum l x m x\right)$, el tiempo generacional $\left(G=\frac{\sum_{x} x l x m x}{R_{x}}\right)$, el valor reproductivo a la edad $\mathrm{x}\left(v_{x}=\frac{\sum_{x}^{\infty} l x m x}{l x}\right)$, la esperanza de vida a la edad $\mathrm{x}\left(e_{x}=\frac{T_{x}}{l x}\right.$, donde $T_{x}=\sum_{x}^{\infty} L x$ es el tiempo por vivir y $L x=t(l x+l x+1) / 2$ es el tiempo vivido en un intervalo t) y la tasa intrínseca de crecimiento poblacional con el estimador de Laughlin $\left(r=\frac{\ln R_{0}}{G}\right)$.

Con las variables del régimen demográfico: sobrevivencias, $p x$, y fecundidades, $m x$, se construyó la matriz de Leslie:

$L=\left[\begin{array}{cccc}m_{1} & m_{2} & \ldots & m_{18} \\ p_{1} & 0 & \ldots & 0 \\ \ldots & \ldots & \ldots & \ldots \\ 0 & \ldots & p_{17} & 0\end{array}\right]$ y considerandoel vector etario inicial: $N_{0}=\left[\begin{array}{l}n_{1} \\ n_{2} \\ . \\ . \\ n_{18}\end{array}\right]$ se realizó la proyección de la población a 25 años considerando la iteración $N_{t+1}=L \cdot N_{t}$. Como valor inicial se consideraron cuatro arañas adultas de un año y dos de dos años.

\section{Resultados}

En las cohortes 1 y 3 nacieron 79 y 106 arañuelas. En la otra cohorte nació un número excepcionalmente bajo en esta especie: 12 arañuelas. Todas las cohortes actualmente tienen arañas vivas: en 439 días de seguimiento de la cohorte 1 hay una hembra adulta y en 411 días de seguimiento de Cohorte 2 hay también una hembra adulta, obtenidas después de 9-10 mudas. Mientras que en 390 días de seguimiento de la Cohorte 3 hay un macho maduro (sub-adulto), después de 8 mudas (Figura 1). La relación macho: hembra fue difícil de estimar ya que esta sólo se realizó por observación fotográfica considerando sólo las arañuelas con sexo claramente identificable. A los estados 6-7 fue 1: 1,83 $(\mathrm{n}=17)$. Actualmente es 1:2. Las temperaturas y humedades relativas a lo largo del tiempo de observación fueron similares en las tres cohortes (Tabla 1). Los tamaños de los diferentes estadios de desarrollo variaron desde los 2,3 $\mathrm{mm}$ al nacimiento hasta los 12,9 mm en la etapa adulta (Tabla 2, Figura 2). El cambio de estadio de desarrollo se muestra en las Figuras 2 y 3 y el $\mathrm{T}_{50}$ para cada estadio en la Tabla 3 .

La tabla de vida (Tabla 4) permitió estimar $R_{0}=2,117$, $G=751,01$ días y $r=0,00099\left(\right.$ días $\left.^{-1}\right)$. La simulación con

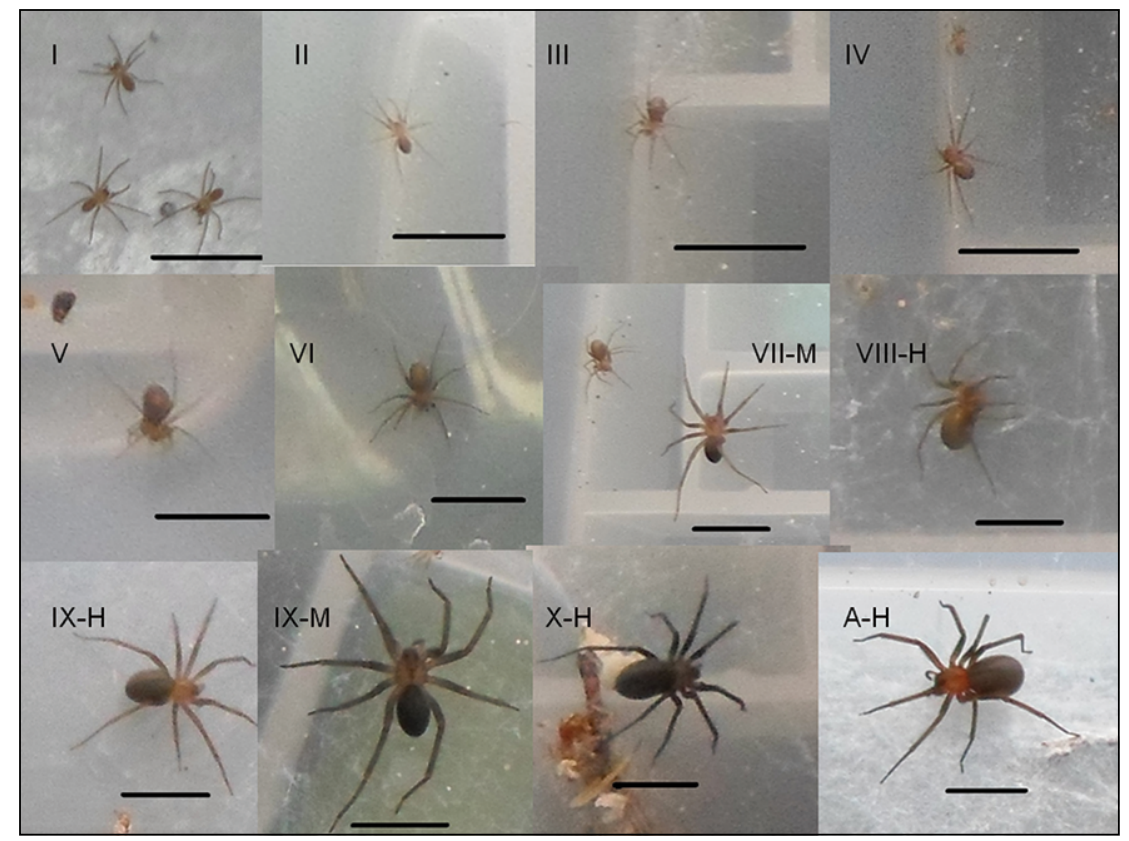

Figura 1. Fotografías de los diferentes estados de desarrollo de Loxosceles laeta. Estados 1 al 10 con números romanos y adulto con la letra $\mathrm{A}$. La clave $-\mathrm{H}$ significa hembra y $-\mathrm{M}$, macho. 
Tabla 1. Temperaturas (T) y humedades relativas (HR) en el interior de las cámaras de crianza para tres cohortes de Loxosceles laeta (C1, C2 y

C3) en las diferentes estaciones durante el período experimental

\begin{tabular}{|lllcc} 
& & \multicolumn{1}{c}{ C1 } & C2 & C3 \\
Verano-2013 & $\mathrm{T}\left({ }^{\circ} \mathrm{C}\right)$ & $24,9 \pm 5,1$ & $25,3 \pm 1,4$ & $25,5 \pm 1,9$ \\
& $\mathrm{HR}(\%)$ & $47,2 \pm 6,1$ & $41,8 \pm 7,1$ & $39,0 \pm 5,8$ \\
Otoño-2013 & $\mathrm{T}\left({ }^{\circ} \mathrm{C}\right)$ & $18,2 \pm 3,5$ & $18,4 \pm 3,9$ & $18,3 \pm 3,9$ \\
& $\mathrm{HR}(\%)$ & $41,0 \pm 0,2$ & $42,1 \pm 0,2$ & $42,7 \pm 0,6$ \\
\multirow{5}{*}{ Invierno-2013 } & $\mathrm{T}\left({ }^{\circ} \mathrm{C}\right)$ & $18,4 \pm 1,5$ & $18,5 \pm 1,5$ & $18,9 \pm 1,6$ \\
& $\mathrm{HR}(\%)$ & $41,2 \pm 3,2$ & $45,4 \pm 2,8$ & $45,9 \pm 2,3$ \\
Primavera-2013 & $\mathrm{T}\left({ }^{\circ} \mathrm{C}\right)$ & $20,8 \pm 2,8$ & $21,3 \pm 1,9$ & $28,1 \pm 2,6$ \\
& $\mathrm{HR}(\%)$ & $44,5 \pm 10,2$ & $41,4 \pm 5,3$ & $40,1 \pm 5,1$ \\
Verano-2014 & $\mathrm{T}\left({ }^{\circ} \mathrm{C}\right)$ & $27,1 \pm 4,9$ & $25,9 \pm 4,7$ & $25,7 \pm 7,6$ \\
& $\mathrm{HR}(\%)$ & $44,5 \pm 6,0$ & $44,6 \pm 6,3$ & $41,9 \pm 9,0$ \\
\hline
\end{tabular}

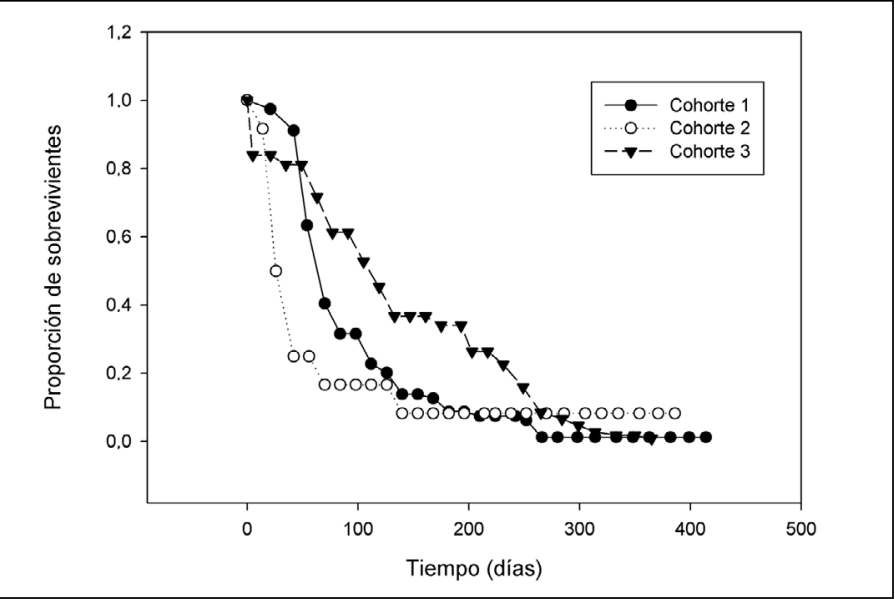

Figura 2. Evolución de la proporción de sobrevivientes en tres cohortes de Loxosceles laeta.

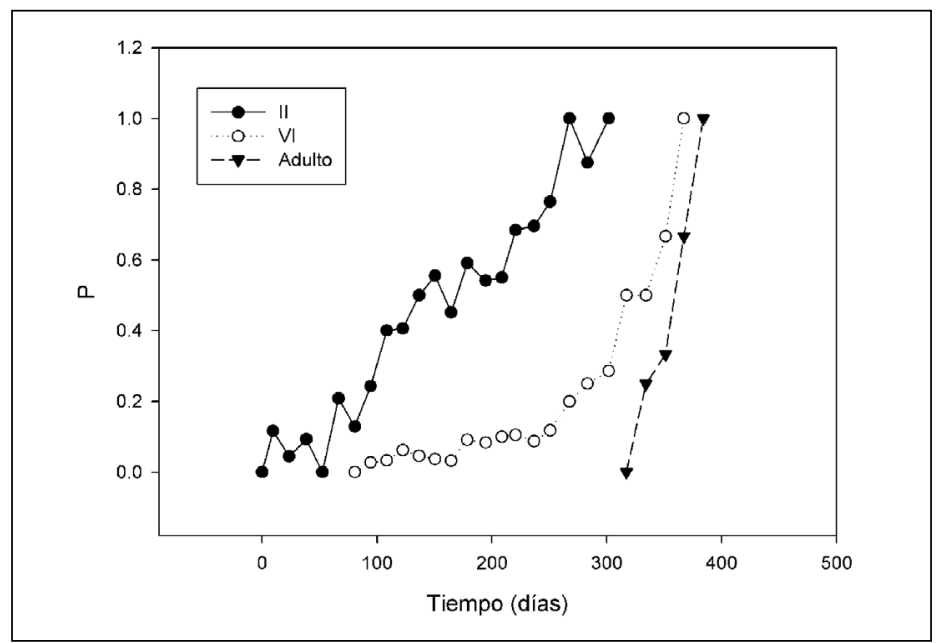

Figura 3. Evolución de la proporción de individuos que alcanzan los estados 2 (II), $6(\mathrm{VI})$ y adulto en tres cohortes de Loxosceles laeta.
Tabla 2. Longitud corporal de los diferentes estados de desarrollo (11 a adulto) de Loxosceles laeta

\begin{tabular}{|ccc|}
\hline Estadio & Tamaño promedio $(\mathbf{m m})$ & Desviación estándar \\
11 & 2,31 & 0,27 \\
12 & 3,10 & 0,19 \\
13 & 3,72 & 0,19 \\
14 & 4,36 & 0,28 \\
15 & 5,25 & 0,18 \\
16 & 6,44 & 0,45 \\
17 & 6,76 & 0,41 \\
18 & 8,04 & 0,47 \\
19 & 9,53 & 0,20 \\
110 & 10,36 & 0,89 \\
Adulto & 12,88 & 0,85 \\
\hline
\end{tabular}

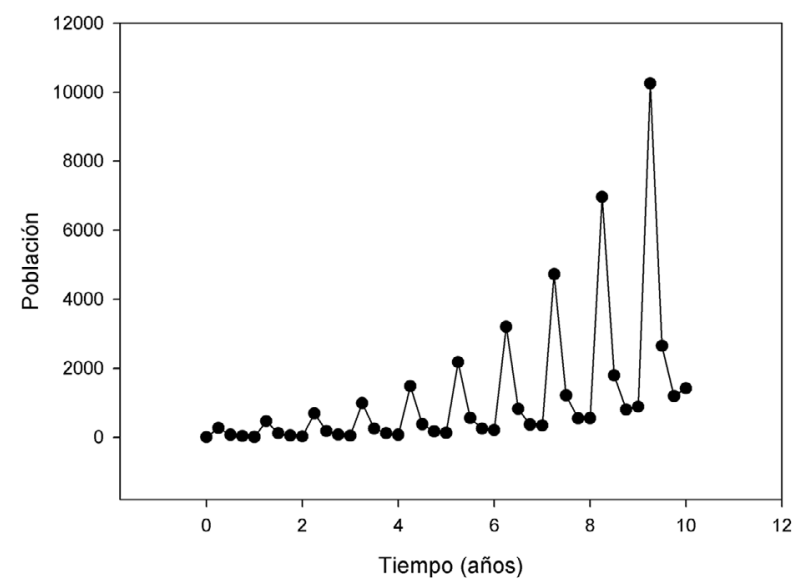

Figura 4. Proyección de una población de Loxosceles laeta a partir de 6 adultos en función del tiempo, con simulación con matriz de Leslie basados en la sobrevivencia $(p x)$ y fecundidad $(m x)$.

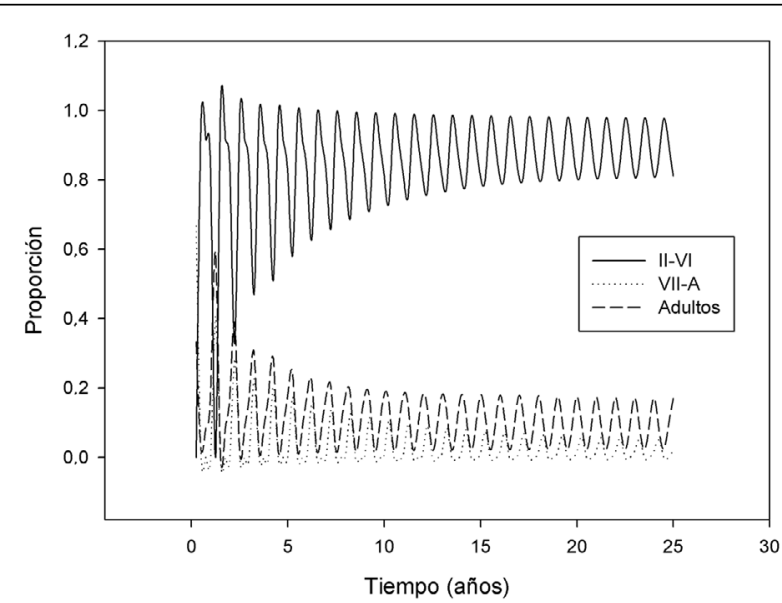

Figura 5. Variaciones en las proporciones de estados inmaduros (I-VI), subadultos (VII-A) y adultos en función del tiempo, con simulación con matriz de Leslie basados en la sobrevivencia $(p x)$ y fecundidad $(m x)$. 
la matriz de Leslie muestra fluctuaciones en el crecimiento poblacional y en las proporciones de estados inmaduros y adultos a lo largo del tiempo (Figuras 4 y 5).

\section{Discusión}

Los capullos de las dos primeras hembras analizadas dieron origen a 79 y 106 arañuelas, lo que da un promedio de 92,5 arañuelas por capullo, hecho que es consistente con lo reportado por Galiano (1967) 34 : 88,4 con un rango entre 22 y 138 arañuelas. También es consistente con lo reportado para otras especies del género. Por ejemplo 50 arañuelas (0-91) para $L$. reclusa $a^{42}, 30$ arañuelas para $L$. intermedia $(30-50)^{42}, 61,3$ arañuelas para L. gaucho $(25-$ $117)^{43}$ y 33,7 arañuelas para $L$. hirsuta ${ }^{42}$. El capullo de una de nuestras hembras dio origen a sólo 12 arañuelas, lo que es excepcionalmente bajo, hecho que podría ser explicado por ejemplo, por tratarse de una hembra envejecida. Las arañuelas de primer estado medían alrededor de $2,3 \mathrm{~mm}$ y se mantuvieron en este estado por largo tiempo, mudando $50 \%$ de ellas al siguiente estado recién a $\operatorname{los} 147 \pm 54$ días, es decir, entre tres y seis meses. El tiempo promedio de desarrollo para llegar al estado adulto fue de $351,7 \pm 7,9$ días en hembras y el hecho que nuestro único macho superviviente tiene 390 días y aún no complete su desarrollo es consistente con lo reportado por $^{34}$ Galiano (1967): 316 días para hembras 406,5 días para machos. También es consistente con lo reportado por Galiano y Hall (1973) ${ }^{24}$ : 328,5 días para hembras y 454,7 días para machos, en Argentina. Sin embargo, este resultado es absolutamente diferente del reportado por Lowrie (1987) ${ }^{26}$ en Los Ángeles (E.U.A.) a temperaturas entre 15 y $20^{\circ} \mathrm{C}$ : 819 y 930 días para hembras y machos, respectivamente. Esta diferencia puede ser explicada por diferencias poblacionales y por las condiciones ambientales de humedad, frecuencia de presas y especialmente por diferencias en la temperatura que en nuestro estudio varió entre 15,7 y $31^{\circ} \mathrm{C}$. La relación macho: hembra fue difícil de evaluar sólo por fotografías y por la alta mortalidad diferencial en los primeros estadios por lo que sólo se tiene el número estimativo 1: 2,81 al estado 6-7. Sin embargo, al estado adulto, aunque sólo sobreviven tres individuos, esta relación es 1:2. Estos resultados son consistentes con lo reportado para otras especies de Loxosceles. Por ejemplo se han reportado índices de 1:1,2 en promedio en L. gaucho pero con variaciones entre cohortes desde 1:7 hasta $1,2: 1^{43}$. En Chile, al estado adulto se ha reportado una relación 1: $8^{19}$, lo que según la evolución de nuestras cohortes podría ser explicado por mortalidad diferencial, ya que los machos sobreviven menos que las hembras. El tamaño final de las arañas es consistente con lo reportado en la literatura científica ${ }^{23}$ y consistente con nuestras propias observaciones donde el tamaño llega a $14 \mathrm{~mm}$, la envergadura puede superar los $4 \mathrm{~cm}$ y donde la masa
Tabla 3. Edad en la cual $50 \%$ de los individuos alcanza un estado particular $\left(T_{50}\right)$ en la araña Loxosceles laeta y su error estándar (ES)

\begin{tabular}{|cccccccc} 
& $\mathbf{b}_{\mathbf{0}}$ & $\mathbf{b}_{\mathbf{1}}$ & $\mathbf{R}^{2}$ & $\mathbf{F}$ & $\mathbf{P}$ & $\mathbf{T}_{50}$ & $\mathbf{E S}$ \\
12 & 2,31 & 0,0183 & 0,65 & 38,84 & 0,0003 & 146,74 & 54,51 \\
13 & 0,92 & 0,0179 & 0,56 & 22,49 & 0,0002 & 227,68 & 55,78 \\
14 & 0,85 & 0,0165 & 0,48 & 16,37 & 0,0009 & 251,03 & 60,47 \\
15 & 1,00 & 0,0145 & 0,57 & 27,32 & 0,0001 & 275,70 & 68,97 \\
16 & 0,59 & 0,0155 & 0,58 & 26,27 & 0,0001 & 284,21 & 64,53 \\
17 & 0,11 & 0,0163 & 0,61 & 28,62 & 0,0001 & 298,52 & 61,02 \\
18 & $-2,98$ & 0,026 & 0,65 & 24,63 & 0,0003 & 306,56 & 38,37 \\
19 & $-6,53$ & 0,036 & 0,67 & 21,15 & 0,0013 & 318,14 & 27,58 \\
\hline 110 & $-19,88$ & 0,074 & 0,76 & 20,42 & 0,0020 & 338,54 & 13,60 \\
Adulto & $-39,52$ & 0,127 & 0,84 & 22,21 & 0,0180 & 351,66 & 7,89 \\
\hline
\end{tabular}

B0 y B1 representan el intercepto y la pendiente en la regresión con el modelo probito, $R^{2}$ el coeficiente de determinación, $\mathrm{F}$ el valor del estadígrafo y $\mathrm{P}$ el $\mathrm{p}$-valor.

\begin{tabular}{|c|c|c|c|c|c|c|c|c|c|c|c|}
\hline$x$ & $N x$ & Ix & $m x$ & $d x$ & $q x$ & $p x$ & Ixmx & $V x$ & $L x$ & $T x$ & $e x$ \\
\hline 91,25 & 197 & 1 & 0 & 0,76 & 0,76 & 0,24 & 0 & 2,11 & 56,51 & 93,33 & 93,33 \\
\hline 182,5 & 47 & 0,239 & 0 & 0,14 & 0,60 & 0,40 & 0 & 8,87 & 15,29 & 36,82 & 154,3 \\
\hline 273,8 & 19 & 0,096 & 0 & 0,08 & 0,84 & 0,16 & 0 & 21,95 & 5,095 & 21,54 & 223,3 \\
\hline 365 & 3 & 0,015 & 45 & 0 & 0 & 1 & 0,69 & 139 & 1,39 & 16,44 & 1080 \\
\hline 456,3 & 3 & 0,015 & 0 & 0 & 0 & 1 & 0 & 94 & 1,39 & 15,05 & 988,5 \\
\hline 547,5 & 3 & 0,015 & 0 & 0 & 0 & 1 & 0 & 94 & 1,39 & 13,66 & 897,3 \\
\hline 638,8 & 3 & 0,015 & 0 & 0 & 0 & 1 & 0 & 94 & 1,39 & 12,27 & 806 \\
\hline 730 & 3 & 0,015 & 45 & 0 & 0 & 1 & 0,69 & 94 & 1,39 & 10,89 & 714,8 \\
\hline 821,3 & 3 & 0,015 & 0 & 0 & 0 & 1 & 0 & 49 & 1,39 & 9,50 & 623,5 \\
\hline 912,5 & 3 & 0,015 & 0 & 0 & 0 & 1 & 0 & 49 & 1,39 & 8,11 & 532,3 \\
\hline 1004 & 3 & 0,015 & 0 & 0 & 0 & 1 & 0 & 49 & 1,39 & 6,72 & 441 \\
\hline 1095 & 3 & 0,015 & 45 & 0,01 & 0,33 & 0,67 & 0,69 & 49 & 1,158 & 5,33 & 349,8 \\
\hline 1186 & 2 & 0,01 & 0 & 0 & 0 & 1 & 0 & 6 & 0,926 & 4,17 & 410,6 \\
\hline 1278 & 2 & 0,01 & 0 & 0 & 0 & 1 & 0 & 6 & 0,926 & 3,24 & 319,4 \\
\hline 1369 & 2 & 0,01 & 0 & 0 & 0 & 1 & 0 & 6 & 0,926 & 2,32 & 228,1 \\
\hline 1460 & 2 & 0,01 & 6 & 0,01 & 0,50 & 0,50 & 0,06 & 6 & 0,695 & 1,39 & 136,9 \\
\hline 1551 & 1 & 0,005 & 0 & 0 & 0 & 1 & 0 & 0 & 0,463 & 0,70 & 136,9 \\
\hline 1643 & 1 & 0,005 & 0 & 0,01 & 1 & 0 & 0 & 0 & 0,232 & 0,23 & 45,63 \\
\hline
\end{tabular}

En esta Tabla $x$ corresponde a la marca de clase del intervalo de edad $x, n x$ es el número de sobrevivientes a la edad $x, I x$ la proporción de sobrevivientes, $d x$, la proporción de muertos en el intervalo, $q x$ la probabilidad de muerte, $p x$ la probabilidad de sobrevivir en ese intervalo (sobrevivencia), $m x$ la fecundidad, $v x$ el valor reproductivo, $L x$ el tiempo vivido en el intervalo $x$, Tx el tiempo por vivir y ex la esperanza de vida a la edad $x$. 
promedio de los machos es de $105,21 \pm 48,75 \mathrm{mg}$ y de las hembras $238,82 \pm 66,14 \mathrm{mg}^{33}$.

En las tablas de vida se consideró la sobrevivencia constante a partir del primer año para decaer en el último año de vida, lo cual es consistente con una variación de menos de $10 \%$ en la sobrevivencia de adultos reportados para esta especie ${ }^{26}$, lo que le da realidad al modelo. La curva de sobrevivencia fue de tipo III, con alta mortalidad en juveniles ${ }^{44}$. La sobrevivencia constante de adultos puede ser explicada por el hecho que estando en sus microambientes preferentes, con presas disponibles, como pequeñas moscas, grillos y otras presas, accesibles en los rincones sin acceso a aseo, y sin predadores, la probabilidad de morir es baja. Las fecundidades consideradas al primer, segundo y tercer año corresponden a la mitad de los huevos de un capullo promedio en esta especie porque sólo se consideró la fracción hembra de la población y se supuso una relación macho: hembra $=1: 1$. Este supuesto puede ser considerado algo irreal ya que, por ejemplo, en adultos se han reportado relaciones $1: 8^{19}$. Sin embargo, es posible que la relación de sexos asimétrica se vaya estableciendo por mortalidad diferencial de los machos a lo largo del desarrollo. La relación 1:1 supuesta es consistente con la encontrada, 1:1,2, para L. gaucho ${ }^{43}$ por lo que aunque conservadora, no es irreal. Como era de esperar, el valor reproductivo de una hembra, que representa el valor relativo de una hembra de cierta edad en cuanto a sus posibilidades de contribución poblacional a través de su progenie, llega a un máximo al año cuando la hembra es adulta y tiene elevadas probabilidades de sobrevivencia y reproducción. Se mantiene relativamente alto el segundo y tercer año, decayendo en el último período de vida. La esperanza de vida al nacer es baja, sólo de tres meses, lo que es explicado por la alta mortalidad inicial, lo que es característico de especies r-estrategas que tienen elevado número de crías, con escaso o nulo cuidado parental ${ }^{44}$. Posteriormente la esperanza de vida se eleva en la medida que se superan las barreras de mortalidad. Hasta alcanzar su máximo al año. La tasa reproductiva neta fue sólo moderada $^{2,12}$ aunque superior a lo reportado para otras arañas, por ejemplo 1,4 para Diguetia mojavea ${ }^{45}$ y superior a algunos insectos como la vinchuca Triatoma infestans: $1,36^{38}$. Esto conduce a una tasa de incremento también moderada a baja $\left(r=0,00099\right.$ días $\left.^{-1}\right)$. El tiempo generacional fue estimado en un valor razonable de alrededor de dos años (751,01 días), superior a otras arañas ${ }^{45}$.

La proyección de la población con matrices de Leslie a partir de un valor inicial de seis arañas adultas con estos parámetros poblacionales indican que el crecimiento poblacional de estas arañas seguiría un incremento con fuertes fluctuaciones estacionales producidos por el fenómeno reproductivo en los meses de alto calor (diciembreenero-febrero), lo que es consistente con las observaciones de terreno. Además se muestra que a partir del cuarto o quinto año, la población total puede superar los miles y por tanto haber cientos de adultas. Estos valores son sólo referenciales ya que el modelo usado no supone conflictos de espacio, ni supone competencia o denso dependencia. Sin embargo, los estudios realizados en terreno dan cuenta de una gran infestación domiciliaria, por ejemplo $40,6 \%$ de las casas urbanas y $24,4 \%$ de las casas rurales reportadas en $1970^{19}$. Aunque estos datos son antiguos y no se han realizado nuevos estudios para ver posibles cambios, una prevalencia razonable de infestación es 1 de cada 3 casas, lo que es consistente con lo reportado por Schenone $(1970)^{19}$, en promedio $29 \%$. Una infestación tan grande se puede obtener por permanencia de estados adultos y dispersión de las formas inmaduras. Por otra parte, que en una casa haya decenas o cientos de arañas también es consistente con el promedio de 3,9 arañas por casa en áreas urbanas y 11,9 en áreas rurales, con un máximo de $163 \pm 56$ arañas/casa en las cinco casas más infestadas reportadas en los estudios de Schenone ${ }^{19}$. Datos actuales en áreas urbanas Kansas en 2004 reportan un promedio de $83,5 \pm 114,9$ arañas/casa de L. reclus $a^{46}$.

Un resultado muy interesante a partir de la simulación es que por las características del régimen demográfico, nuestro modelo predice fluctuaciones en las proporciones relativas de estados inmaduros vs maduros, inducidos por la reproducción estacional. Como es de esperar, en período reproductivo hay gran número de arañuelas y pocas adultas. Nuestras simulaciones muestran que los estados 1 a 6 , es decir, pequeñas arañuelas inmaduras menores a 6,5 milímetros pueden constituir 80 a $90 \%$ de la población. En 1970, Schenone y cols., encontraron $79,6 \%$ de adultas y $20,4 \%$ restante de inmaduras. Este dato no parece muy razonable pues implicaría una pirámide poblacional invertida, contraria al alto número de arañuelas por capullo, y puede explicarse fácilmente por la dificultad que ofrece encontrar e identificar las arañuelas más pequeñas. Nuestros datos sugieren que si el promedio de 3,9 arañas adultas/casa en área urbana es correcto, la población real con formas inmaduras y maduras, fluctuaría entre 20 arañas en época no-reproductiva y 40 arañas en época reproductiva. Este es un dato eco-epidemiológico interesante que sugiere que si se observa una araña en la casa, hay que considerar toda la casa infestada con una población mucho mayor.

La regulación de la población de L. laeta es importante por su potencial peligro. Esta especie, con su alto potencial reproductivo, puede mantener y dispersar grandes poblaciones. La regulación por depredación por la araña tigre, Scytodes globula, es insuficiente para erradicar a L. laeta ${ }^{33}$ por lo que junto a las medida de no eliminar a la araña tigre, hay que insistir en las medidas de control tales como el aseo de la casa y los closets y el cuidado, limpieza y vigilancia de la ropa, sábanas y toallas, especialmente en el crepúsculo y noche y en temporadas cálidas. 
Agradecimientos. Los autores agradeces el financiamiento de la investigación al Proyecto Fondecyt 1110058.

\section{Resumen}

Introducción: A pesar del abundante conocimiento eco-epidemiológico de la araña del rincón, Loxosceles laeta, causante de todos los cuadros de loxoscelismo en Chile, sigue siendo una especie poco conocida en el ambiente médico, especialmente las características y tiempos de sus estados de desarrollo. Objetivo: En este estudio abordamos estos aspectos con el objetivo de aportar imágenes claras del desarrollo de esta especie y por primera vez de sus características y proyecciones poblacionales y de la relación entre estados maduros e inmaduros, datos útiles para el control y prevención de los accidentes por mordedura. Resultados: Loxosceles laeta es una especie r-estratega, con un $R_{0}=2,1$, un tiempo generacional $G=2,1$ años y con concentración del valor reproductivo de las hembras entre el primer y segundo año de vida. Determinamos los tamaños promedio y los tiempos de desarrollo de todos los estados. Los primeros varían entre los $2,3 \mathrm{~mm}$ al nacer y alrededor de $13 \mathrm{~mm}$ al estado adulto. Los tiempos totales de desarrollo son de aproximadamente un año. Discusión: La proyección de la población con matriz de Leslie sugiere una gran capacidad de crecimiento y dispersión, pero con claras fluctuaciones poblacionales estacionales asociadas a la reproducción. Además se muestra que la proporción de estados inmaduros fluctúa estacionalmente entre $80 \mathrm{y}$ $90 \%$, lo que implica que una casa con tres o cuatro arañas adultas visibles, en realidad tiene una población entre 20 y 40 arañas en total.

\section{Referencias bibliogáficas}

1.- Reyes H, Noemí I, Gottlieb B. Arácnidos y otros artrópodos ponzoñosos. En Atías A (ed): Parasitología Clínica: 1991; Mediterráneo, Santiago, Chile, pp. 553-65.

2.- Zavaleta A. Loxoscelismo, un problema de salud en el Perú. Bol OPS 1987; 103: 378-86.

3.- Macchiavelo A. La Loxosceles laeta, causa del aracnoidismo cutáneo o mancha gangrenosa en Chile. Rev Chil Hist Nat 1937; 41: 11-9.

4.- Schenone H, Reyes H. Animales ponzoñosos de Chile. Bol Chil Parasitol 1965; 20: 104-9.

5.- Atkins J A, Wingo C W, Sodeman W A. Probable cause of necrotic spider bites in the Midwest. Science 1957; 126: 73.

6.- Casanueva M E. Los licosidos de Chile. Estudio biológico y taxonómica por los métodos de sistemática alfa y taxonomía numérica (Araneae: Lycosidae). Gayana 1980; 42: 5-76.

7.- Donoso-Barros R. Consideraciones sobre aracnoidismo cutáneo en Chile. Arch Ur Med Cir Esp 1949; 32: 184-206.

8.- Schenone H. Loxoscelismo cutáneo de predominio edematoso. Bol Chil Parasitol 1998; 53: 78-83.

9.- Schenone H. Cuadros tóxicos producidos por mordedura de araña en Chile: latrodectismo y loxoscelismo. Rev Med Chile 2003; 131: 437 44.

10.- Schenone H. A propósito del loxoscelismo en Chile. Rev Med Chile 2004; 132: 121-2.

11.- Canals M, Casanueva M E, Aguilera M. Cuáles son las especies de arañas peligrosas en Chile? Rev Med Chile 2004; 132: 773-6.

12.- Canals M, Casanueva M E, Aguilera M E. Arañas y escorpiones. 2008. En Zoología Médica II. Invertebrados. (Canals M Cattan PE, Eds). Editorial Universitaria, Santiago, Chile, pp. 145-83.

13.- Canals M. Biología e historia natural de la araña del rincón Loxosceles laeta. Parasitol Día 2011; 1: 4-5.

14.- Manríquez J J, Silva S. Loxoscelismo cutáneo y cutáneo-visceral. Rev Chilena Infectol 2009; 26: 420-32.

15.- Platnick N I. The world spider catalog. The American Museum of Natural History, Merrett P \& HD Cameron Eds, New York 2004.

16.- Ríos J, Pérez M, Sánchez M, Bettini M, Mieres J, Paris E. Caracterización clínicoepidemiológica telefónica de la mordedura por araña de rincón en un centro de información toxicológica de Chile, durante el año 2005. Rev Med Chile 2007; 135: 1160-5.

17.- Schenone H, Rubio S, Saavedra S, Rojas A. Loxoscelismo en pediatría: Región Metropolitana, Chile. Rev Chil Pediatr 2001; 72: $1-8$.

18.- Fernández D, Ruz L, Toro H. Aspectos de la biología de Scytodes globula Nicolet, 1949 (Aranae: Scytodidae), un activo depredador de Chile Central. Acta Entomol Chil 2002; 26: 17 25.

19.- Schenone H, Rojas A, Reyes H, Villarroel F, Suárez G. Prevalence of Loxosceles laeta in houses in central Chile. Amer J Trop Med Hyg 1970; 19: 564-7.

20.- Villarroel F, Schenone H, Rojas A, Sanhueza H. Distribution by stage of development and sex of Loxosceles laeta caught in the central zone of Chile. Bol Chil Parasitol 1971; 26: 59-60.

21.- Alfaro C, Veloso C, Torres-Contreras H, Solís R, Canals M. 2013. Thermal niche overlap of the spider of the nook Loxosceles laeta (Araneae; Sicariidae) and its possible predator, the tiger spider Scytodes globula (Scytodidae). J Therm Biol 2013; 38: 502-7.
22.- Canals, M, Alfaro C, Veloso C, TorresContreras, H, Solís R. 2013. Tolerancia a la desecación y sobreposición del nicho térmico entre la araña del rincón Loxosceles laeta y un posible control biológico, la araña tigre Scytodes globula. Parasitología Ibero-Latinoamericana 72 (1): 52-60.

23.- Parra D, Torres M, Morillas J, Espinoza P. Loxosceles laeta, identificación y una mirada bajo microscopía de barrido. Parasitol Latinoam 2002; 57: 75-8.

24.- Galiano M E, Hall M. Datos adicionales sobre el ciclo vital de Loxosceles laeta (Nicolet) (Araneae). Physis 1973; 32: 277-88.

25.- Schenone H, Letonja T. Notas sobre la biología y distribución geográfica de las arañas del género Loxosceles. Bol Chil Parasitol 1975; 30: 27-9.

26.- Lowrie D C. Effects of diet on the development of Loxosceles laeta (Nicolet) (Araneae, Loxoscelidae). J Arachnol 1987; 15: 303-8.

27.- Vetter R. Spider of the genus Loxosceles (Araneae; Sicariidae): a review of biological, medical and physiological aspects regarding envenomations. J. Arachnol 2008; 36: 150-63.

28.- Schenone H, Saavedra T, Rojas A, Villarroel F. Loxoscelismo en Chile. Estudios epidemiológicos, clínicos y experimentales. Rev Inst Med Trop Sao Paulo. 1989; 31: 403-15.

29.- Levi H W, Spielman A. The biology and control of the South American brown spider Loxosceles laeta (Nicolet) in a north American focus. Amer J Trop Med Hyg 1964; 13: 132-6.

30.- Gilbert C, Rayor L S. Predatory behavior of spitting spiders (Araneae, Scytodidae) and the evolution of prey wrapping. J Arachnol 1985; 13: 231-41.

31.- Bowden K. The evolution of sociality in the spitting spider Scytodes fusca (Araneae, 
Scytodidae)-evidence from observations of intraespecific interactions. J Zool 1991; 223: 161-72.

32.- Canals M, Solís R. Is the tiger spider Scytodes globula an effective predator of the brown recluse spider Loxosceles laeta? Rev Med Chile 2013; 141: 805-7.

33.- Canals M, Arriagada N, Solís R. Interactions between the Chilean recluse spider Loxosceles laeta (Araneae; Sicariidae) and the araneophagic spitting spider Scytodes globula (Scytodidae). J Med Entomol (in press).

34.- Galiano M E. Ciclo biologico e desarrollo de Loxosceles laeta (Nicolet, 1849). Acta Zool Lill 1967; 23: 431-64.

35.- Gertsch W J. The spider genus Loxosceles in South America (Araneae, Scytodidae) Bull Amer Mus Nat Hist 1967; 136: 117-74.

36.- Gertsch W J, Ennik F. The spider genus Loxosceles in North America, Central America and the West Indies (Araneae, Loxoscelidae).
Bull Amer Mus Nat Hist 1983; 175: 264-360.

37.- Canals M, Cattan P E, Ehrenfeld M. Algunas estimaciones numéricas de la importancia epidemiológica de los vectores de la enfermedad de Chagas en Chile. Parasitol Día 1993; 17: 7986.

38.- Canals M, Cattan P E, Valderas J, Solís R. Efectos poblacionales de fluctuaciones de mortalidad y fecundidad en Triatoma infestans: Simulación mediante matrices de Leslie. Rev Med Chile 1991; 119: 1239-42.

39.- Canals M, Cattan P E, Ehrenfeld M, Torres P. Poblaciones experimentales de Triatoma infestans: Efectos de condiciones ambientales variables. Parasitol Día 1992; 16: 72-7.

40.- Canals M, Cattan P E, Ehrenfeld M. Sobrevivencia de Triatoma spinolai en ambiente habitacional. Parasitol Día 1994; 18: 82-7.

41.- Ehrenfeld M, Canals M, Cattan P E. Population parameters of Triatoma spinolai under different environmental conditions and densities. J Med
Entomol 1998; 35: 740-4

42.- Fisher M L, Marquez Da Silva E. Comportamiento sexual de L. hirsuta Melo Leitao 1934 (Araneae, Sicariidae). Rev Etol 2001; 2: 31-42.

43.- Rinaldi I M P, Forti L C. Stropa A A. On the development of the brown spider Loxosceles gaucho Gertsch (Araneae, Sicariidae). Rev Bras Zool 1997; 14: 697-706.

44.- Fuentes E. 1989. Ecología: Introducción a la teoría de poblaciones y comunidades. Ediciones Universidad Católica de Chile. Santiago.

45.- Boulton A M, Polis G A. Phenology and life history of the desert spider, Diguetia mojavea (Araneae, Diguetidae). J Arachnol 1999; 27 : 513-21.

46.- Sandidge J. Predation by cosmopolitan spiders upon the medically significant pest species Loxosceles reclusa (Araneae, Sicariidae): limited possibilities for biological control. J Econ Entomol 2004; 2: 230-4. 